\title{
The Determination of Vehicle Speeds from Delta-V in Two Vehicle Planar Collisions
}

\author{
J Neades AiTS, A5 Lakeside Business Park, South Cerney, Gloucestershire, UK \\ R Smith Faculty of Technology, De Montfort University, Leicester, UK.
}

\begin{abstract}
The change of a vehicle's velocity, delta-V $(\Delta v)$, due to an impact is often calculated and used in the scientific investigation of road traffic collisions. In isolation however, this figure does not yield any information concerning the actual velocities of the vehicles and such information is often of prime concern to those investigating collisions. In this paper a method is developed which uses the change in velocity sustained by a vehicle in a planar collision to estimate the velocities of the vehicle before and after a collision. The key equations are derived from conservation of momentum, conservation of energy and restitution. As with the calculation of delta- $\mathrm{V}$, the method requires an initial estimate of the principal directions of force. The pre and post impact angles of the vehicles' velocities can be used to obtain better estimates of the principal directions of force and of the coefficient of restitution. In collisions where it is difficult to analyse the vehicles' post-impact motion, this method provides a way to estimate the actual speeds of vehicles. To demonstrate the method, it is used to analyse one of the RICSAC collisions. The results of an analysis of other staged collisions illustrate the accuracy of the method.
\end{abstract}

Keywords: speed change, velocity change, vehicle collisions

\section{INTRODUCTION}

The calculation of vehicle speeds is of prime importance to courts. Such reconstructions have traditionally centred on the analysis of tyre and other marks on the road surface, see for example, by Smith $[\mathbf{1}, \mathbf{2}]$ There are a variety of methods that provide information on vehicle speeds in the absence of tyre marks. One such method involves the use of the pedestrian throw distance discussed, for example, by Evans and Smith [3]. Another method which does not rely on tyre marks is discussed here. Essentially this method uses the change in velocity of each vehicle to determine the total closing speed of the vehicles. From the total closing speed it is then possible to derive the pre and post impact velocities. This method is not limited to any particular method by which the changes in velocity are generated. So it can be used as well with in-car accident data recorders and with other impact phase models such as CRASH3.

The CRASH3 algorithm is commonly used to establish the change in velocity of the vehicles. A description of the CRASH3 algorithm is provided by Day and Hargens [4] where they outline a PC version of the model (EDCRASH). There are various other PC versions available. For example AiDamage (by Neades) [5] is the de facto standard implementation in 
the UK. It is used by over $85 \%$ of police forces in the UK and by organisations such as Loughborough University's Vehicle Safety Research Centre and the Transport Research Laboratory. Other models, such as the Planar Impact Mechanics (PIM) model described by Brach [6] and a similar derivation by Ishikawa [7] are also useful in collision reconstruction. These are essentially forward iterative models and do not directly predict pre-impact velocities. Smith [8] has derived and generalised the CRASH formulae based on a general planar impact model.

Models for the impact phase of collisions commonly make a number of assumptions. These assumptions are also adopted here. First tyre and other external forces are assumed to be negligible during the impact, so that momentum is conserved. Second, the vehicle masses and moments of inertia are maintained throughout the collision. That is the deformations caused by the collision do not significantly change the moments of inertia and the masses of the vehicles are not significantly changed, for example, by parts of a vehicle becoming detached as a result of the collision. Third, the time-dependent impulse is modelled by one force, its resultant, which acts at some point on or in the vehicles. The discussion here is restricted to two vehicle planar collisions. For collisions involving significant vertical motion, this analysis will need modification.

\section{PLANAR COLLISIONS}

Rose et al [9] use a heuristic method based on McHenry's spring model [4] to obtain some interesting and helpful results for collisions. In this section, Smith's [8] analysis is extended along the lines of Rose et al to provide expressions for the change in velocity of the vehicles along the line of action of the impulse. The changes in velocity of the vehicles tangentially to the line of action of the impulse are then considered. These changes is velocity are precursors in determining the total closing speed of the vehicles which is central to this work. The analysis presented in this section provides a more rigorous and general basis for the results. However, more importantly, this analysis generalises and extends the results of Rose et al to include the effects of restitution.

With the assumptions outlined previously, conservation of momentum and the definition of velocity change gives

$$
\begin{aligned}
& m_{1} \boldsymbol{u}_{1}+m_{2} \boldsymbol{u}_{2}=m_{1} \boldsymbol{v}_{1}+m_{2} \boldsymbol{v}_{2}, \\
& \Delta \boldsymbol{v}=\boldsymbol{v}-\boldsymbol{u}
\end{aligned}
$$

Equations (1) and (2) lead to

$$
\Delta \boldsymbol{v}_{1}=-\Delta \boldsymbol{v}_{2} \frac{m_{2}}{m_{1}}
$$


A diagram showing a generalised impact configuration is shown in Figure 1. (For clarity, the two vehicles are shown separated.)

\section{Fig 1.}

\section{Collision Configuration}

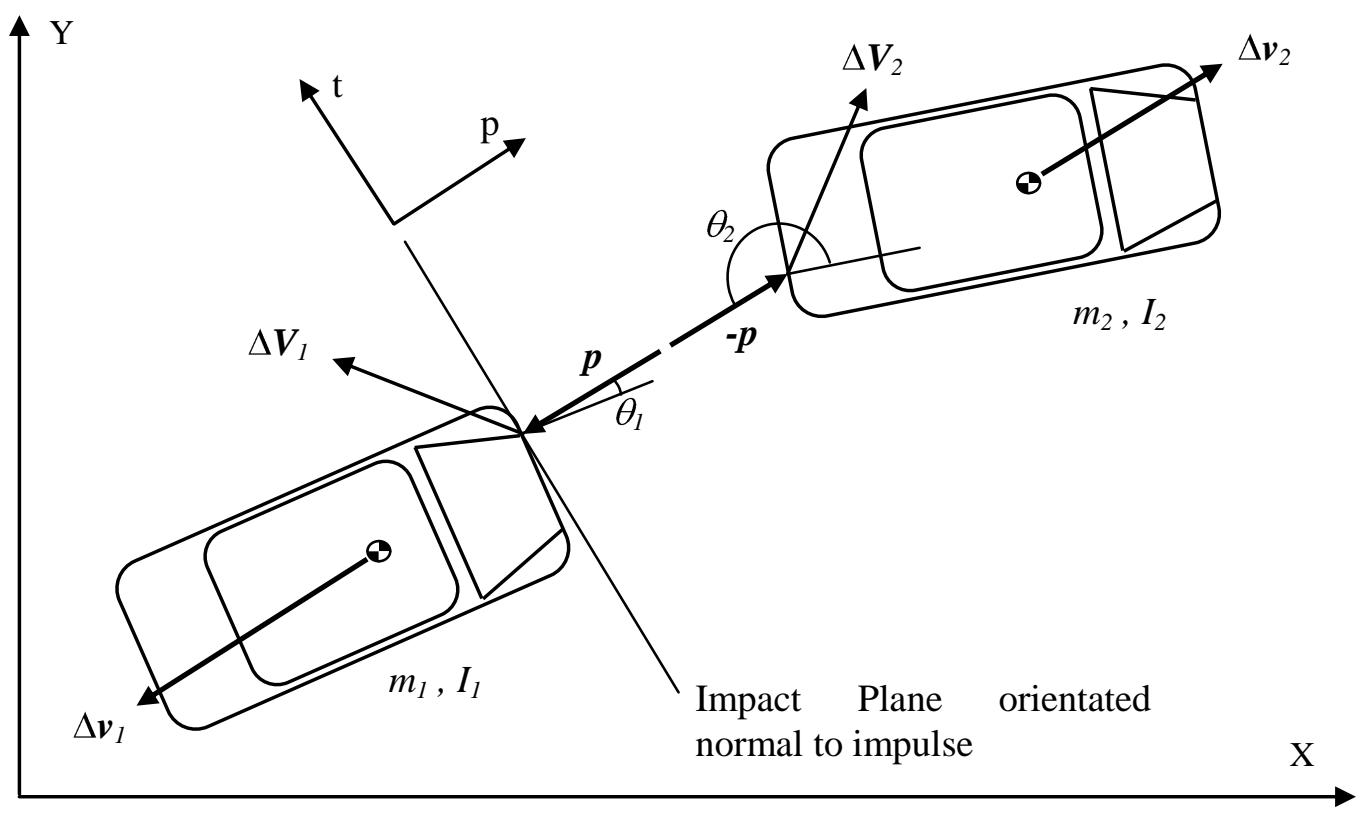

In collinear collisions, the line of action of the impulse $\boldsymbol{P}$ passes through the centres of mass of the vehicles and there is no change in the rotational velocity of either vehicle. If $\boldsymbol{P}$ does not act through the centres of mass it produces a change not only in the motion of the centres of mass, but also a rotation of each vehicle about the centre of mass given by

$$
P h=I(\Omega-\omega)=m k^{2} \Delta \omega
$$

where $k$ is the radius of gyration of the vehicle and $h$ is the perpendicular distance from the centre of mass of the vehicle to the line of action of the impulse $\boldsymbol{P}$. The change in rotation $(\Delta \omega)$ produced on each vehicle as a result of the impact can now be described using equation (5)

$$
\Delta \omega_{1}=\frac{h_{1}}{k_{1}^{2}} \Delta v_{1}, \quad \Delta \omega_{2}=\frac{h_{2}}{k_{2}^{2}} \Delta v_{2}
$$

Lower case symbols are used for motion at the centre of mass. Upper case symbols are used here to distinguish motion at the point of application of the impulse so that $U_{\mathrm{p}}$ denotes the component of the vehicle's velocity before impact in the direction of $\boldsymbol{p}$ at the point where the impulse $\boldsymbol{P}$ acts then 


$$
U_{1 \mathrm{p}}=\boldsymbol{u}_{1} \cdot \boldsymbol{p}+h_{1} \omega_{1}, \quad U_{2 \mathrm{p}}=\boldsymbol{u}_{2} \cdot \boldsymbol{p}+h_{2} \omega_{2},
$$

where $\boldsymbol{p}$ is a unit vector in the direction of $\boldsymbol{P}$ and the subscript p denotes that this component applies along the line of action of the impulse. Similarly $V_{\mathrm{p}}$ denotes the component of vehicle's velocity after impact in the direction of $\boldsymbol{p}$

$$
V_{1 \mathrm{p}}=\boldsymbol{v}_{1} \cdot \boldsymbol{p}+h_{1} \Omega_{1}, \quad V_{2 \mathrm{p}}=\boldsymbol{v}_{2} \cdot \boldsymbol{p}+h_{2} \Omega_{2} .
$$

The coefficient of restitution $\left(e_{\mathrm{p}}\right)$ for the vehicles in the direction of $\boldsymbol{P}$ at the point where the impulse acts gives

$$
V_{2 \mathrm{p}}-V_{1 \mathrm{p}}=-e_{\mathrm{p}}\left(U_{2 \mathrm{p}}-U_{1 \mathrm{p}}\right)
$$

Substitute equations (2) and (3) together with (6) to (8) into equation (1) to give

$$
m_{2}\left(1+e_{\mathrm{p}}\right)\left(U_{2 \mathrm{p}}-U_{1 \mathrm{p}}\right)=\left(m_{1}+m_{2}\right) \Delta v_{1}-m_{1} h_{1} \Delta \omega_{1}-m_{2} h_{2} \Delta \omega_{2}
$$

From equations (3) and (5) it follows that

$$
\Delta v_{1}=\frac{m_{2}\left(1+e_{\mathrm{p}}\right)\left(U_{2 \mathrm{p}}-U_{1 \mathrm{p}}\right)}{\left(m_{1} \delta_{2}+m_{2} \delta_{1}\right)}
$$

where

$$
\delta=1+\frac{h^{2}}{k^{2}}
$$

Result (10) gives the changes in velocity at the centre of mass of the vehicle in terms of the pre-impact closing speed of the points of contact between the vehicles. Since the closing speed of the vehicles is unknown for the majority of collisions, such a result is of limited use. However the closing speed is related to the energy lost as a result of the collision in the form of crush damage. The work done in causing crush can be estimated with the methods described by Day and Hargens [4] or any other suitable method, such as Brach's PIM [6]. Smith [8] writes the total energy absorbed by the vehicles as a result of the collision as the sum of the translational energy, $E_{\mathrm{T}}$, and rotational energy, $E_{\mathrm{R}}$, lost so

$$
E=E_{\mathrm{T}}+E_{\mathrm{R}}
$$


The use of equations (2) and (3) lead to

$$
E_{\mathrm{T}}=m_{1} \Delta v_{1}\left(\boldsymbol{u}_{2} \cdot \boldsymbol{p}-\boldsymbol{u}_{1} \cdot \boldsymbol{p}\right)-\frac{1}{2} m_{1}\left(\Delta v_{1}\right)^{2}\left(1+\frac{m_{1}}{m_{2}}\right)
$$

and equations (4) and (5) yield

$$
E_{\mathrm{R}}=m_{1} \Delta v_{1}\left(h_{2} \omega_{2}-h_{1} \omega_{1}\right)-\frac{1}{2} m_{1}\left(\Delta v_{1}\right)^{2}\left(\frac{h_{1}^{2}}{k_{1}^{2}}+\frac{m_{1} h_{2}^{2}}{m_{2} k_{2}^{2}}\right) .
$$

Equation (12) can be solved for the closing speed component to give

$$
U_{2 \mathrm{p}}-U_{1 \mathrm{p}}=\frac{E}{m_{1} \Delta v_{1}}+\frac{\Delta v_{1}\left(m_{1} \delta_{2}+m_{2} \delta_{1}\right)}{2 m_{2}}
$$

The substitution of $U_{2 \mathrm{p}}-U_{1 \mathrm{p}}$ into equation (10) leads to the commonly used formula

$$
\Delta v_{1}=\sqrt{\frac{2 E m_{2}\left(1+e_{\mathrm{p}}\right)}{m_{1}\left(m_{1} \delta_{2}+m_{2} \delta_{1}\right)\left(1-e_{\mathrm{p}}\right)}} .
$$

(Note that in Smith [8] $\delta_{1}$ and $\delta_{2}$ were inadvertently transposed in several of the equations.) When $e_{\mathrm{p}}$ is zero this equation gives the CRASH3 algorithm. Smith [8] has extended the CRASH3 algorithm to include the effects of restitution which are shown in equation (16). This together with equation (3) are key equations in the calculation of the speed changes from the energy absorbed by the damage. These are staging posts in the calculation of the closing speeds and so of the vehicles speed. Equations (10) and (16) both describe the change in velocity at the centre of mass $(\Delta v)$ along the line of action of the impulse. From equations (6) and (7) the change in velocity at the point of application of the impulse $\left(\Delta V_{\mathrm{p}}\right)$ in the direction of $\boldsymbol{p}$ is given by

$$
\Delta V_{\mathrm{p}}=\Delta v \cdot \boldsymbol{p}+h \Delta \omega
$$

The substitution of equations (5) and (11) into equation (14) produces

$$
\Delta V_{1 \mathrm{p}}=\delta_{1}\left(\Delta \boldsymbol{v}_{1} \cdot \boldsymbol{p}\right), \quad \Delta V_{2 \mathrm{p}}=\delta_{2}\left(\Delta \boldsymbol{v}_{2} \cdot \boldsymbol{p}\right)
$$

Result (18) shows that along the line of action of the impulse $\boldsymbol{P}$, the change in velocity of the point of application is equal to the product of the change in velocity at the centre of mass and the scalar value delta. 
In addition to the change in velocity along the line of action of the impulse there is also a tangential change in velocity at the points of action $\left(\Delta V_{t}\right)$ due to the consequent change in rotation as defined by equation (5). Use the subscript $t$ to denote motion in a direction perpendicular to the line of action of the impulse. If $U_{\mathrm{t}}$ and $V_{\mathrm{t}}$ are used to denote the component of the vehicle's velocity before impact in a direction perpendicular to $\boldsymbol{p}$ at the point where the impulse $\boldsymbol{P}$ acts then

$$
U_{\mathrm{t}}=|\boldsymbol{u} \times \boldsymbol{p}|+h_{\mathrm{t}} \omega, \quad V_{\mathrm{t}}=|\boldsymbol{v} \times \boldsymbol{p}|+h_{\mathrm{t}} \Omega
$$

where $h_{\mathrm{t}}$ is the distance from the point of application of the impulse to the centre of mass and

$$
d^{2}=h^{2}+h_{\mathrm{t}}^{2}
$$

From Newton's laws of motion there can be no change in velocity at the centre of mass perpendicular to the impulse $\boldsymbol{P}$. So any change in velocity of the points of action tangential to the impulse can only be due to a change in the angular velocity of the vehicle. Thus

$$
\Delta V_{1 \mathrm{t}}=h_{1 \mathrm{t}} \Delta \omega_{1}, \quad \Delta V_{2 \mathrm{t}}=h_{2 \mathrm{t}} \Delta \omega_{2}
$$

\section{CLOSING SPEEDS}

In this section the closing speed parallel and perpendicular to the impulse is derived and used to determine the total closing speed of the two vehicles. Equation (10) may be used to obtain the closing speed parallel to the impulse or substitute equation (16) into equation (10) to obtain

$$
U_{2 \mathrm{p}}-U_{1 \mathrm{p}}=\sqrt{\frac{2 E\left(m_{1} \delta_{2}+m_{2} \delta_{1}\right)}{m_{1} m_{2}\left(1-e_{\mathrm{p}}^{2}\right)}} .
$$

When $e_{\mathrm{p}}$ is zero this equation gives the result of Rose et al [9]. It has extended their formula for the component of the closing speed in the direction of the PDOF to include the effects of restitution. This is a key equation in the calculation of the closing speed and so the vehicle speeds.

The tangential component change in velocity at the point where the impulse acts is given by equation (21). It follows that

$$
\Delta V_{2 \mathrm{t}}-\Delta V_{1 \mathrm{t}}=h_{2 \mathrm{t}} \Delta \omega_{2}-h_{1 \mathrm{t}} \Delta \omega_{1}
$$


Substitute equations (3), (5) and (19) into equation (23) to yield

$$
\Delta V_{2 \mathrm{t}}-\Delta V_{1 \mathrm{t}}=-m_{1} \Delta v_{1}\left[\frac{h_{1 \mathrm{t}} h_{1}}{m_{1} k_{1}^{2}}+\frac{h_{2 \mathrm{t}} h_{2}}{m_{2} k_{2}^{2}}\right]
$$

In many collisions the surface of the vehicles do not slide over each other or finish sliding before the contact ends. For such collisions

$$
V_{2 \mathrm{t}}=V_{1 \mathrm{t}}
$$

so that equation (24) becomes

$$
U_{2 \mathrm{t}}-U_{1 \mathrm{t}}=m_{1} \Delta v_{1}\left[\frac{h_{1 \mathrm{t}} h_{1}}{m_{1} k_{1}^{2}}+\frac{h_{2 \mathrm{t}} h_{2}}{m_{2} k_{2}^{2}}\right] .
$$

This formula gives the component of the closing speed perpendicular to the direction of the PDOF. It complements the result of Rose et al [9]. This formula gives this component in terms of $\Delta v$ and so includes the effects of restitution via equation (16). This is a key equation in the calculation of the closing speeds and so of the vehicles speed. The total closing speed $\left(U_{\mathrm{R}}\right)$ can now be expressed as the vector sum of the components determined by results $(10)$ or (22) and (26)

$$
U_{\mathrm{R}}=\sqrt{U_{2 \mathrm{p}}-U_{1 \mathrm{p}}^{2}+U_{2 \mathrm{t}}-U_{1 \mathrm{t}}{ }^{2}} .
$$

The angle of the closing speed vector to the impulse $\boldsymbol{P}(\beta)$ is

$$
\tan \beta=U_{2 t}-U_{1 \mathrm{t}} / U_{2 \mathrm{p}}-U_{1 \mathrm{p}}
$$

Specifically

$$
\tan \beta=\frac{m_{1} m_{2}}{\left(m_{1} \delta_{2}+m_{2} \delta_{1}\right)}\left[\frac{h_{1 \mathrm{t}} h_{1}}{m_{1} k_{1}^{2}}+\frac{h_{2 \mathrm{t}} h_{2}}{m_{2} k_{2}^{2}}\right]\left(1+e_{\mathrm{p}}\right) .
$$

As $e_{\mathrm{p}}$ increases so does $\beta$.

CRASH3 calculations usually require the principal direction of force (PDOF) for each vehicle; this is the direction in which the impulse acts. The impact geometry is illustrated in Figure 2 where two vehicles V1 and V2 collide obliquely as shown in the insert. 


\section{Fig 2.}

\section{Impact Geometry}

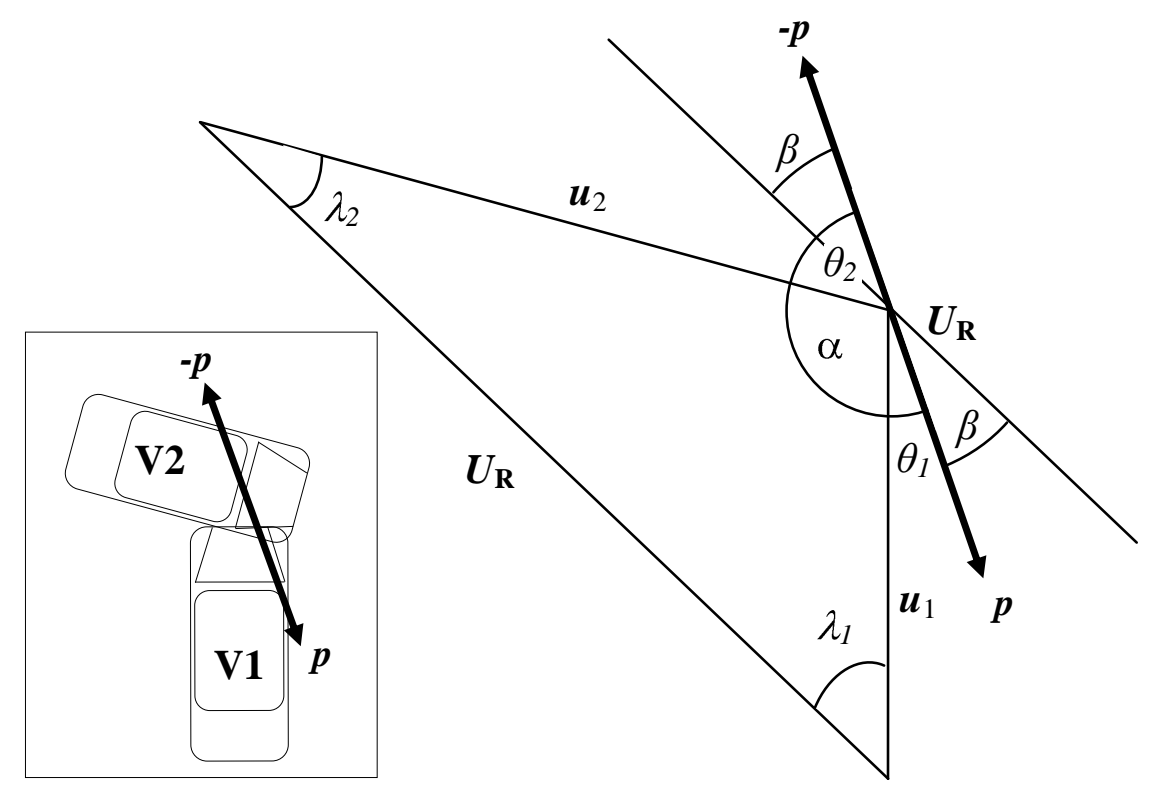

It follows that the angle between the two vehicles at impact, $\alpha$, is related to the PDOF, $\theta$, by

$$
\alpha=\pi-\theta_{1}-\theta_{2}
$$

and that the angle, $\lambda$, between the initial heading of vehicles and the closing speed satisfy

$$
\lambda_{1}=\beta+\theta_{1}, \quad \lambda_{2}=\pi-\alpha-\lambda_{1}=\theta_{2}-\beta .
$$

The absence of significant pre-impact rotation is a common feature in many collisions. In such cases the closing velocity of the points of action for each vehicle will also be the closing velocity of their centres of mass. (If there is pre-impact rotation, additional information will be required to resolve the difference between the velocity at these two points and the method outlined here will need to be extended.) The Sine Rule gives

$$
U_{2}=u_{2}=\frac{U_{\mathrm{R}} \sin \lambda_{1}}{\sin \alpha}, \quad U_{1}=u_{1}=\frac{U_{\mathrm{R}} \sin \left(\lambda_{2}\right)}{\sin \alpha}
$$

\section{DISCUSSION}

The method developed here leading to result (32) can be used with any model which gives the velocity changes of each vehicle. Any force-crush model can be used to calculate the energy absorbed. The method can also be used with results from in-car accident data recorders. A model commonly used to generate velocity changes is CRASH3 which uses the 
damage to the vehicles to obtain the energy absorbed. It uses a linear force-crush model. (See e.g. Day and Hargens [4] or McHenry [10]). Practical considerations for measuring vehicles are described more fully by Neades and Shephard [11]. The centroid of the damage is often used to define the points of application of the impulse and the shape of the damage is used to estimate the PDOF. Ishikawa [7] proposes a method whereby the impact centre is taken to be the mid-point of the contacting surfaces at the point of maximum deformation. The PDOF is then assumed to lie along a line perpendicular to the line of the contacting surfaces through the impact centre. The difference between these two methods is usually small and the choice of PDOF is discussed further in section 6.

In the majority of substantial vehicle to vehicle collisions, the points of application of the impulse reach a common tangential velocity, hence the assumption of a common tangential velocity in equation (25). If the coefficient of restitution in the direction of the impulse is also zero then the points of application of the impulse reach a common velocity during the collision phase. This is the common velocity assumption present in many of the CRASH3 derivations. As described previously, Smith [8] shows that the common velocity assumption may be relaxed somewhat by the inclusion of a non-zero coefficient of restitution along the line of action of the impulse. This leads to equation (16) which can be viewed as an extension to the standard or zero restitution CRASH3 model. If the coefficient of restitution in the direction of the impulse is greater than zero, then the points of application of the impulse reach a common velocity along the line of action of the impulse at the moment of maximum engagement. At the moment of maximum engagement the maximum amount of energy has been absorbed by the vehicle structures. If energy is then returned to the vehicles due to restoration of the vehicle structure, the velocities of the vehicles continue to change beyond that required simply to reach a common velocity at the point of application of the impulse as outlined by Brach [6].

Smith and Tsongas [12] report a series of staged collisions where they found that the coefficient of restitution was between 0 and 0.26. In general, they report that lower values of restitution tend to be found as the closing speed increases. Wood [13] also suggests a similar relationship based on a series of full scale crash tests with a maximum restitution of about 0.3 More recently Rose, Fenton and Beauchamp [14] investigated the effects of restitution for a single type of vehicle in head-on collisions with a barrier. They found coefficients of restitution from 0.11 to 0.19 for impact speeds around $47-57 \mathrm{kmh}^{-1}$. Cipriani et al [15] studied a series of vehicle to vehicle collinear impacts with low speeds up to $7 \mathrm{~ms}^{-1}$ and obtained values from about 0.2 to 0.6 with the lower values found for higher impact speeds.

The use of a positive coefficient of restitution $e_{\mathrm{p}}$ increases the component closing speeds which are determined by equations (22) and (26). In turn this leads to a larger total closing speed calculated by equation (27). An increase in the coefficient of restitution tends therefore to increase the pre-impact speeds determined by equation (32) for each vehicle. Minimum pre-impact speeds are therefore calculated when $e_{\mathrm{p}}$ is zero, which is likely to be close to the actual value for higher speed collisions. However the minimum impact speed is often of prime importance in criminal investigations. 
Once the pre-impact velocities are found from equation (32) then the velocity change determined by equation (16) can be used to calculate the post-impact velocity for each vehicle. In real-world collisions the impact configuration together with the post-impact directions of travel of the centre of mass are often known although the speed after impact may not be known. This suggests a method of refining any initial estimate of the PDOF values so that the predicted post-impact directions of travel match those recorded for the actual collision. This method leads to an estimate of the coefficient of restitution along the line of the impulse. It is outlined below.

\section{EXAMPLE COLLISIONS}

The model presented here was used with data from the Research Input for the Computer Simulation of Automobile Collisions full scale tests (RICSAC) [16]. Several authors have analysed the RICSAC tests in detail and a number of discrepancies between those analyses are apparent e.g. Smith and Noga [17] and Brach [18]. In several of the tests there are significant discrepancies between the recorded damage profiles and the photographs of the damage. These discrepancies result in very large force differences in the calculations. This is particularly evident in tests 2, 6 and 7 where force differences of $469 \%, 577 \%$ and $608 \%$ respectively were obtained. Appendix B details the collision type and force differences obtained.

Test 8 of the series was a set up to be representative of a $90^{\circ}$ intersection collision with both vehicles travelling at $9.2 \mathrm{~ms}^{-1}$ at impact. A CRASH3 damage analysis shows that with the PDOF values as recorded, the work done in causing deformation to the vehicles was $63 \mathrm{~kJ}$. Using the recorded PDOF values and a zero coefficient of restitution, the method described here uses equation (16) to determine the speed change in the direction of the PDOF. Equation (22) gives the closing speed in the direction of the impulse as $12.83 \mathrm{~ms}^{-1}$. Equation (26) gives the closing speed perpendicular to the impulse as $5.86 \mathrm{~ms}^{-1}$. These component results can be used in equation (27) to determine the total closing speed as $14.1 \mathrm{~ms}^{-1}$. With this configuration the angle $\lambda_{1}$ is $24.5^{\circ}$ and angle $\alpha$ is $90^{\circ}$. Using equation (32) the preimpact speeds are found to be $8.18 \mathrm{~ms}^{-1}$ for vehicle 1 and $11.49 \mathrm{~ms}^{-1}$ for vehicle 2 . From these values and the calculated changes in velocity from equation (16) the post-impact motion can be determined using equation (2).

Diagrams in Jones and Baum [16] show that for Test 8 the centres of mass of each vehicle moved off along a common post-impact direction of approximately $40^{\circ}-50^{\circ}$ to the original direction of travel of vehicle 1 . The calculated post-impact motion of the vehicles for Test 8 with a zero coefficient of restitution shows that the centres of mass of the vehicles do not follow the recorded post-impact direction of travel. Indeed when the coefficient of restitution is close to zero the vehicles appear to pass through each other as shown in the first part of Figure 3. This cannot be a realistic scenario for this type of impact configuration. A more realistic model can be achieved however by using a non-zero coefficient of restitution $e_{\mathrm{p}}$. The post-impact motion predicted for RICSAC Test 8 using coefficients of restitution of 0.0 
and 0.3 are shown in Figure 3 to illustrate this effect. The PDOF for each vehicle and the coefficient of restitution are difficult to determine accurately. Various reasonable values were tried and the best ones selected on the basis of the force balance and post-impact direction of travel. The optimum values gave pre-impact speeds of $8.9 \mathrm{~ms}^{-1}$ for vehicle 1 and $9.0 \mathrm{~ms}^{-1}$ for vehicle 2 which underestimate the measured speeds by 0.3 and $0.2 \mathrm{~ms}^{-1}$ respectively.

Fig 3.

\section{RICSAC Test 8: Motion of Centres of Mass with varying coefficients of restitution}

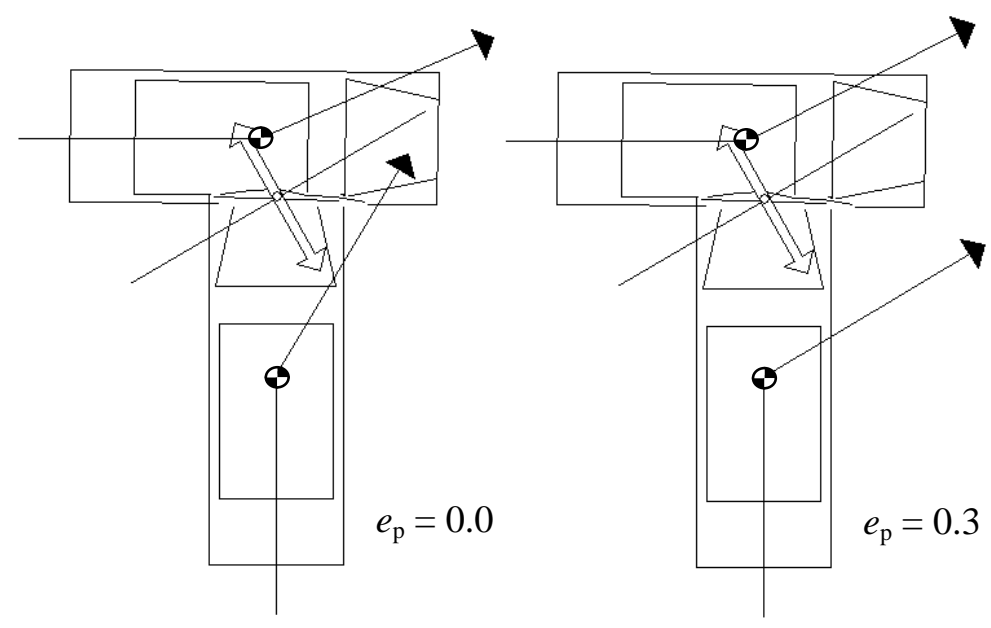

The remainder of the RICSAC tests can be treated in a similar way to calculate pre-impact speeds for these tests. Early versions of the CRASH measuring protocols indicated that crush damage should be measured at the level of maximum intrusion. Later versions of CRASH suggest that crush damage should be measured at the main load bearing level, i.e. at bumper and sill level. This is detailed further in Neades and Shephard [11]. Comparison between the photographs and the recorded measurements suggest that the early measurement version was used to determine the damage profiles. For example the photographs of vehicle 2 in both tests 1 and 2 show considerable intrusion at about mid-door level but much less intrusion at sill level. One author (Neades) has examined and measured scores of damaged vehicles. Based on this experience, photographs and the measurements an estimate of the likely crush at the load bearing level have been made for each vehicle. The adjustments made vary dependent on the particular damage to each vehicle. Although such a process is somewhat rough and ready the resulting measurements provide a better approximation of the damage profiles to the stiff parts of the vehicles.

In addition the PDOF values for each vehicle were adjusted so that although the configuration of the vehicles at impact remained constant, the post-impact directions of travel for the centres of mass matched those recorded for each of the tests as shown in the diagrams presented by Jones and Baum [16]. Three $90^{\circ}$ impact tests were conducted (Tests, 8, 9 and 10). As outlined previously in each of these collisions a coefficient of restitution of 0.3 has been applied so that a reasonable match was achievable with the recorded post-impact 
motion. Note that using a coefficient of 0.3 produces a reasonable match for each of these three tests. Further adjustment around 0.3 can produce a marginally closer fit but with little change in the calculated closing speed. Details of the adjustments applied for this analysis are shown in Appendices C and D.

The results from this analysis are shown in Appendix E. A graph summarising these results comparing the measured pre-impact speed of each vehicle with the pre-impact speed calculated by this method is shown in Figure 4. (Note that the stationary target vehicles used in tests 3, 4 and 5 have been omitted from the results.)

\section{Fig 4.}

RICSAC Tests: Graph showing percentage difference of pre-impact speed with calculated pre-impact speed

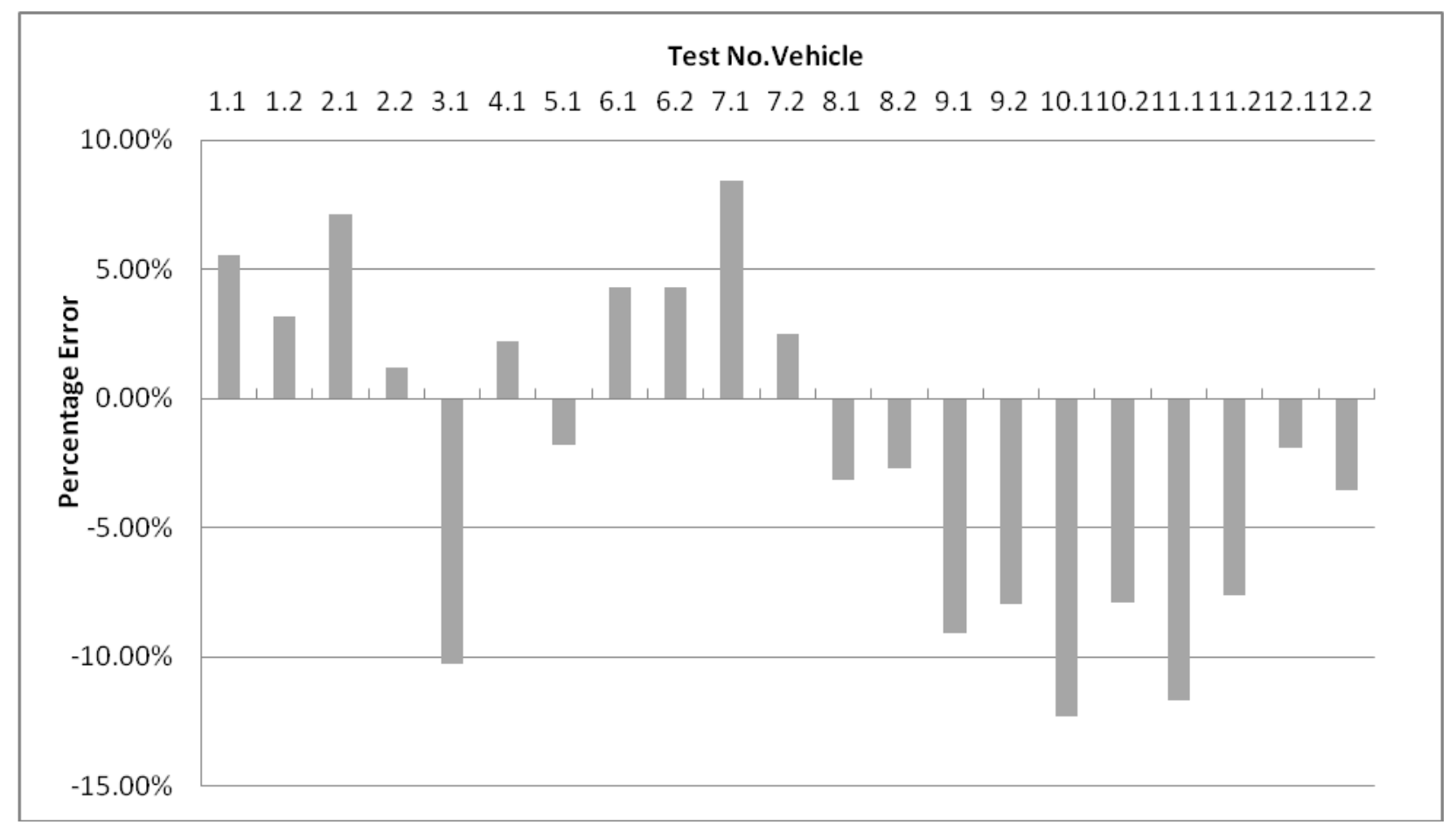

These results indicate that the pre-impact speeds calculated using this technique for the RICSAC tests range from $-12 \%$ to $+8 \%$ with a mean underestimate of $2 \%$. Smith and Noga [19] note that in the collisions they considered, CRASH3 tended to underestimate $\Delta v$ with a mean error of $\pm 13.8 \%$ for higher speed collisions $\left(40-48 \mathrm{kmh}^{-1}\right)$ and $\pm 17.8 \%$ for lower speed collisions $\left(16-24 \mathrm{kmh}^{-1}\right)$. The results here seem also to indicate that the work done in causing crush has been underestimated. One source of error may be that in several of the RICSAC collisions the crush damage profile recorded does not seem to replicate the crush profile as shown in photographs. Although the damage profiles were adjusted in this analysis to better replicate the damage profiles, with more representative measurements a better correspondence to the actual speeds is to be expected. 
In the Lotus crash tests [20] vehicles were crashed into stationary target vehicles. A similar analysis of the crash data as performed for the RICSAC tests reveals a correspondence of calculated impact speeds to actual speeds of between $-9.6 \%$ to $+3.7 \%$ Smith $[8]$ notes that the calculation of $E$ from experimental data is not very precise and that in practical tests it is difficult to separate out the sources of error.

\section{ACCURACY}

Three parameters are identified as key values affecting the overall accuracy of the method and each is considered in turn. These are the impact angle alpha, the method used to determine $\Delta v$ and the choice of the point through which the impulse acts.

With $\alpha$ at 0 or $180^{\circ}$ there is a singularity in result (32). Essentially the collision is one, not two dimensional. In this case equations (1), (2), (8) and (16) can be solved to give the preimpact speeds. At angles close to 0 or $180^{\circ}$, any results from result (32) will be sensitive to the exact angle and should therefore be treated with caution.

The most important factor which affects the accuracy of the calculations are the inaccuracies in the method used to determine the change in velocity itself. If CRASH3 is used to generate $\Delta v$ values the overall accuracy will be broadly similar to those inherent when using CRASH3. However techniques to improve the accuracy of those calculations have been developed and outlined in this paper, such as the inclusion of a coefficient of restitution in as shown by equation (16). The measurements of the damage are critical. However techniques already exist to address poor measurements as outlined above. Implicit in the overall accuracy is the estimation of the direction of the impulse (PDOF) and thereby the angle $\alpha$. In CRASH3 this choice will also affect directly the calculation of energy absorbed by each vehicle. The estimation of the direction of the impulse determines the proportion of the closing speed allocated to each vehicle. Thus an accurate choice is important. Figure 5 shows how the initial speeds of the vehicles are affected by varying the PDOF. Data from RICSAC Test 9 is used together with a zero coefficient of restitution. It is also assumed that the attitude of the vehicles remains constant throughout the impact.

The sensitivity of the results to the actual direction of the impulse as indicated by Figure 5 suggests that the normal visual estimation of the PDOF may not be sufficiently precise. Investigators commonly estimate the direction of the impulse from the pattern of damage sustained by each vehicle. In real-world collisions the immediate post-impact directions of motion of each vehicle can often be deduced from an analysis of tyre and other marks on the roads surface. The techniques described here can then be used to refine the initial estimate of the PDOF and restitution values so that the calculated post-impact directions of travel match those recorded for actual collisions. 
Fig 5.

RICSAC Test 9. Variation of initial vehicle speeds with PDOF

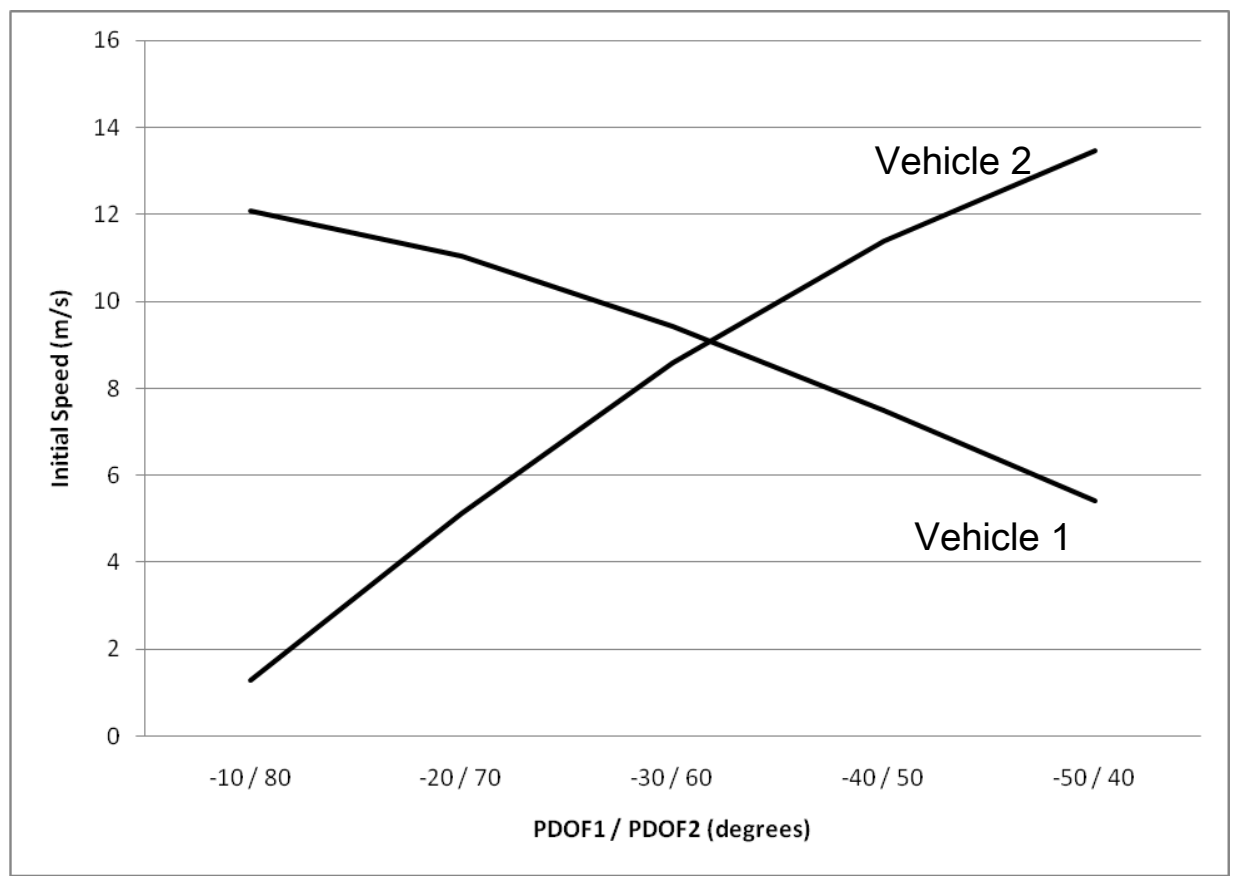

The value of $\Delta v$ is dependent on the value $h$ for each vehicle since this factor not only determines the change in velocity of the centre of mass, but also determines the change in rotation $\Delta \omega$. This value is itself dependent upon the point chosen as the point through which the impulse acts. In CRASH3 calculations the point through which the impulse acts is normally assumed to be the centroid of the damaged area. Ishikawa [7] proposes a method whereby the impact centre is assumed to be the mid-point of the contacting surfaces at the point of maximum deformation. He provides a method whereby that point can be calculated. Unfortunately this calculation requires knowledge of the impulse and post-impact rotation which are themselves affected by the location of this point. It is apparent however that the position of this point could vary by as much as half the crush depth. An initial analysis based on the RICSAC tests produce changes less than $1 \mathrm{~ms}^{-1}$ for each vehicle. This suggests that the calculation of the initial speeds is not particularly sensitive to variations in this parameter.

\section{CONCLUSIONS}

The method presented here shows how the pre-impact speed of a vehicle can be determined from an analysis of the changes in velocity sustained by each vehicle. This data can be from any suitable algorithm that provides such changes in velocity. The technique has been applied to a series of crash tests where changes in velocity were determined with the commonly used CRASH3 algorithm. A technique has also been developed to improve the accuracy of the estimation of the PDOF and the value of the coefficient of restitution. Application of these techniques should provide more reliable results for crash investigators involved in analysing collisions. 


\section{REFERENCES}

1 Smith, R. Skidding to a Stop, Impact 1(1), 11-12, 1990

2 Smith, R. Critical Speed Motion, Impact 2 (1), 12-14, 1991

3 Smith, R. and Evans, A. K. Vehicle Speed calculation from pedestrian throw distance. Proc IMechE Part D 213, 1999

4 Day, T. D. and Hargens, R . L. An Overview of the way EDCRASH computes Delta-V. SAE paper 860209, 1986

5 AiDamage, Ai Training Services Ltd, http://aitsuk.com, 1996 - 2009

6 Brach, R. M. Vehicle Accident Analysis and Reconstruction Methods. SAE International 2005

7 Ishikawa, I. Impact Center and Restitution Coefficients for Accident Reconstruction. SAE paper 940564, 1994

8 Smith, R. The formula commonly used to calculate velocity change in vehicle collisions. Proc IMechE Part D 212, 1998

9 Rose, N. A. Fenton, S. J and Ziernicki, R. M. An Examination of the CRASH3 Effective Mass Concept. SAE paper 2004-01-1181, 2004

10 McHenry, R. R. CRASH 3 User's Guide and Technical Manual. 1981 DOT-HS-805-732 http://www-nass.nhtsa.dot.gov/NASS/MANUALS/Crash3Man.pdf

11 Neades, J. and Shephard, R. Review of Measurement Protocols Applicable to Speed from Damage Programs, Impact 17 (1) 4-12, 2009

12 Smith, R. A. and Tsongas, N. G. Crash Phase Accident Reconstruction. SAE Paper 860209,1986

13 Wood, D. Structural Rebound Characteristics of the Car Population in Frontal Impacts. SAE paper 2000-01-0461, 2000

14 Rose, Nathan A. Fenton, Stephen J. and Beauchamp, Gray Restitution Modeling for Crush Analysis: Theory and Validation. SAE paper 2006-01-0908, 2006

15 Cipriani, A. L. Bayan, F. P. Woodhouse, M. L. Cornetto, A. D. Dalton, A. P. Tanner, C. B. Timbario, T. A. and Dererl, E. S. Low Speed Collinear Impact Severity: A Comparison Between Full Scale Testing and Analytical Prediction Tools with Restitution Analysis. SAE paper 2002-01-0540, 2002

16 Jones I. S. and Baum A. S. Research Input for Computer Simulation of Automobile Collisions Volume IV, DOT HS-805040, 1978

17 Smith R.A and Noga J.T. Examples of Staged Collisions in Accident Reconstruction. Highway Collision Reconstruction, ASME, 1980

18 Brach R.M. Energy Loss in Vehicle Collisions. SAE paper 871993, 1987

19 Smith, R. A. and Noga, J. T. Accuracy and Sensitivity of CRASH. SAE paper 821169, 1982

20 Field Day - Lotus $16^{\text {th }}$ October 1994, Results Issue, Impact 4 (3) 67 - 98, 1995 


\section{APPENDIX A}

Notation

$d \quad$ distance of point of action from centre of mass

$e \quad$ coefficient of restitution

$E \quad$ energy absorbed by each vehicle

$h$ perpendicular distance from the vehicle's centre of mass to the line of action of $\boldsymbol{P}$

I yaw moment of inertia

$k \quad$ radius of gyration for each vehicle

$m \quad$ mass of each vehicle

$\boldsymbol{p} \quad$ unit vector in the direction of $\boldsymbol{P}_{1}$

$\boldsymbol{P} \quad$ impulse due to the collision

$\boldsymbol{u}$ linear velocity of the centre of mass of each vehicle before impact

$U$ component of the velocity of the point of action before impact

$v \quad$ linear velocity of the centre of mass of each vehicle after impact

$V \quad$ component of the velocity of the point of action after impact

$\alpha \quad$ angle between vehicles at impact

$\beta \quad$ angle between $\boldsymbol{p}$ and closing velocity vector

$\gamma \quad$ scalar factor $k^{2} /\left(k^{2}+h^{2}\right)$

$\delta \quad$ scalar factor $1+h^{2} / k^{2}$ i.e. $1 / \gamma$

$\lambda \quad$ angle between closing velocity vector and direction of travel of vehicle

$\theta$ principal direction of force

$\varphi \quad$ angle of point of action relative to vehicle heading

$\Delta v \quad$ velocity change at centre of mass due to impact, $\boldsymbol{v}-\boldsymbol{u}$

$\Delta V \quad$ component of the velocity change at the point of action, $V-U$

$\Delta \omega$ change in angular velocity due to the impact, $\Omega-\omega$

$\omega \quad$ angular velocity of the vehicle before impact

$\Omega \quad$ angular velocity of the vehicle after impact

Subscripts

$\mathrm{p} \quad$ motion along the line of action of $\boldsymbol{P}$

$\mathrm{t}$ motion perpendicular or tangential to the line of action of $\boldsymbol{P}$

1 vehicle 1

2 vehicle 2

$\mathrm{R}$ relative value at the point of action of the impulse $\boldsymbol{P}$ 


\section{APPENDIX B}

RICSAC Tests Summary and Force Difference

\begin{tabular}{|c|c|c|c|c|}
\hline \multirow{2}{*}{ Test } & \multirow{2}{*}{ Impact Type } & \multicolumn{2}{|c|}{ Pre-Impact Speed $\left.\mathbf{( m s}^{-\mathbf{1}}\right)$} & \multirow{2}{*}{ Force Difference (\%) } \\
\cline { 3 - 4 } & & $\mathbf{V 1}$ & $\mathbf{V 2}$ & \\
\hline 1 & $60^{\circ}$ front to side & 8.81 & 8.81 & 363 \\
\hline 2 & $60^{\circ}$ front to side & 13.99 & 13.99 & 469 \\
\hline 3 & $10^{\circ}$ front to rear & 9.43 & 0 & 47 \\
\hline 4 & $10^{\circ}$ front to rear & 17.21 & 0 & 99 \\
\hline 5 & $10^{\circ}$ front to rear & 17.66 & 0 & 385 \\
\hline 6 & $60^{\circ}$ front to side & 9.57 & 9.57 & 577 \\
\hline 7 & $60^{\circ}$ front to side & 12.96 & 12.96 & 608 \\
\hline 8 & $90^{\circ}$ front to side & 9.21 & 9.21 & 14 \\
\hline 9 & $90^{\circ}$ front to side & 9.43 & 9.43 & 80 \\
\hline 10 & $90^{\circ}$ front to side & 14.80 & 14.80 & 66 \\
\hline 11 & $10^{\circ}$ front to front & 9.07 & 9.07 & 4 \\
\hline 12 & $10^{\circ}$ front to front & 13.99 & 13.99 & 29 \\
\hline
\end{tabular}




\section{APPENDIX C}

RICSAC Tests Damage Adjustments

\begin{tabular}{|c|l|}
\hline Test & \multicolumn{1}{|c|}{ Damage Adjustments } \\
\hline 1 & v2 subtract $10 \mathrm{~cm}$ from each $\mathrm{C}_{1}$ to $\mathrm{C}_{6}$ \\
\hline 2 & v2 subtract $15 \mathrm{~cm}$ from each $\mathrm{C}_{1}$ to $\mathrm{C}_{6}$ \\
\hline 3 & v1 add $5 \mathrm{~cm}$ to each $\mathrm{C}_{1}$ to $\mathrm{C}_{6} \cdot \mathrm{v} 2$ offset changed to $-50 \mathrm{~cm}$ \\
\hline 4 & v2 subtract $15 \mathrm{~cm}$ from each $\mathrm{C}_{1}$ to $\mathrm{C}_{6}$ \\
\hline 5 & v2 subtract $20 \mathrm{~cm}$ from each $\mathrm{C}_{1}$ to $\mathrm{C}_{6}$ \\
\hline 6 & v2 subtract $15 \mathrm{~cm}$ from each $\mathrm{C}_{1}$ to $\mathrm{C}_{6}$ \\
\hline 7 & v2 subtract $20 \mathrm{~cm}$ from each $\mathrm{C}_{1}$ to $\mathrm{C}_{6}$ \\
\hline 8 & No adjustment \\
\hline 9 & v2 subtract $10 \mathrm{~cm}$ from each $\mathrm{C}_{1}$ to $\mathrm{C}_{6}$ \\
\hline 10 & v2 add $10 \mathrm{~cm}$ to each $\mathrm{C}_{1}$ to $\mathrm{C}_{6}$ \\
\hline 11 & No adjustment \\
\hline 12 & Expand damage length $L$ for both vehicles to $140 \mathrm{~cm}$ \\
\hline
\end{tabular}




\section{APPENDIX D}

RICSAC Tests PDOF Adjustments

\begin{tabular}{|c|c|c|c|c|}
\hline \multirow{2}{*}{ Test } & \multicolumn{2}{|c|}{ Original values } & \multicolumn{2}{c|}{ Adjusted values } \\
\cline { 2 - 5 } & $\mathbf{V 1}$ & $\mathbf{V 2}$ & $\mathbf{V 1}$ & $\mathbf{V 2}$ \\
\hline 1 & -30 & 30 & -11.3 & 48.7 \\
\hline 2 & -30 & 30 & -11.7 & 48.3 \\
\hline 3 & 0 & 170 & 14.1 & -175.9 \\
\hline 4 & -0.5 & 170.5 & 11.1 & -178.9 \\
\hline 5 & 0 & 170 & 11.6 & -178.4 \\
\hline 6 & -30 & 30 & -11 & 49 \\
\hline 7 & -30 & 30 & -12.7 & 47.3 \\
\hline 8 & -30 & 60 & -19 & 71 \\
\hline 9 & -30 & 60 & -21.8 & 68.2 \\
\hline 10 & -65 & 25 & -25.3 & 64.7 \\
\hline 11 & 4.5 & -4.5 & -2.9 & -11.9 \\
\hline 12 & 4.5 & -4.5 & 1 & -8 \\
\hline
\end{tabular}




\section{APPENDIX E}

RICSAC Results (All values in $\mathrm{ms}^{-1}$ )

\begin{tabular}{|c|c|c|c|c|c|c|c|}
\hline \multirow[t]{2}{*}{ Test } & \multicolumn{2}{|c|}{$\begin{array}{c}\text { Calculated } \\
\text { Delta-V }\end{array}$} & \multirow{2}{*}{$\begin{array}{c}\text { Total Closing } \\
\text { Speed }\end{array}$} & \multicolumn{2}{|c|}{$\begin{array}{l}\text { Measured Pre- } \\
\text { impact Speed }\end{array}$} & \multicolumn{2}{|c|}{$\begin{array}{l}\text { Calculated Pre- } \\
\text { impact Speed }\end{array}$} \\
\hline & V1 & V2 & & V1 & V2 & V1 & V2 \\
\hline 1 & 5.3 & 7.9 & 15.9 & 8.8 & 8.8 & 9.3 & 9.3 \\
\hline 2 & 8.4 & 12.6 & 25.2 & 14.0 & 14.0 & 14.8 & 14.8 \\
\hline 3 & 3.0 & 4.8 & 8.0 & 9.4 & 0.0 & 8.5 & 0.1 \\
\hline 4 & 6.6 & 10.3 & 17.9 & 17.2 & 0.0 & 17.6 & 0.2 \\
\hline 5 & 5.9 & 10.7 & 17.3 & 17.7 & 0.0 & 17.3 & -0.4 \\
\hline 6 & 5.2 & 8.5 & 17.3 & 9.6 & 9.6 & 10.0 & 10.0 \\
\hline 7 & 6.1 & 13.2 & 24.0 & 13.0 & 13.0 & 13.9 & 13.9 \\
\hline $8^{*}$ & 6.6 & 6.2 & 12.6 & 9.2 & 9.2 & 8.9 & 9.0 \\
\hline $9^{*}$ & 6.7 & 3.1 & 12.2 & 9.4 & 9.4 & 8.6 & 8.6 \\
\hline $10^{*}$ & 10.9 & 5.3 & 18.6 & 14.8 & 14.8 & 13.1 & 13.2 \\
\hline 11 & 9.7 & 6.1 & 16.3 & 9.1 & 9.1 & 8.0 & 8.4 \\
\hline 12 & 16.0 & 11.1 & 27.1 & 13.6 & 14.0 & 13.7 & 13.7 \\
\hline
\end{tabular}

